\title{
Radiofrequency Ablation of Idiopathic Left Anterior Fascicular Tachycardia
}

\author{
FRANK BOGUN, M.D., RAFEL El-ATASSI, M.D., EMILE DAOUD, M.D., \\ K. CHING MAN, D.O., S. ADAM STRICKBERGER, M.D., \\ and FRED MORADY, M.D.
}

From the Department of Internal Medicine, Division of Cardiology, The University of Michigan Medical Center, Ann Arbor, Michigan

\begin{abstract}
Left Anterior Fascicular Tachycardia. Introduction: A 45-year-old man with idiopathic ventricular tachycardia (VT) having a right bundle branch block configuration with right-axis deviation underwent an electrophysiologic test.

Methods and Results: Mapping demonstrated a site on the anterobasal wall of the left ventricle where there was an excellent pace map and an endocardial activation time of $-\mathbf{2 0} \mathbf{m s e c}$, but radiofrequency catheter ablation at this site was unsuccessful. At a nearby site, a presumed Purkinje potential preceded the QRS complex by 30 msec during VT and sinus rhythm, and catheter ablation was effective despite a poor pace map and an endocardial ventricular activation time of zero.

Conclusion: Idiopathic VT with a right bundle branch configuration and right-axis deviation may originate in the area of the left anterior fascicle. A potential presumed to represent a Purkinje potential may be more helpful than endocardial ventricular activation mapping or pace mapping in guiding ablation of this type of VT. (J Cardiovasc Electrophysiol, Vol. 6, pp. 1113-1116, December 1995)
\end{abstract}

Purkinje potential, pace mapping, ventricular tachycardia

\section{Introduction}

Idiopathic left ventricular tachycardia (VT) with a right bundle branch block configuration and leftaxis deviation has been demonstrated to arise from the left posterior fascicle. ${ }^{1}$ While a previous study suggested that idiopathic left VT with right-axis deviation may originate in the left anterior fascicle ${ }^{2}$ evidence supporting this possibility has been lacking. The purpose of the present case report is to demonstrate that idiopathic left VT may arise from the area of the left anterior fascicle.

\section{Case Presentation}

A 45-year-old man was referred to the University of Michigan Medical Center for manage-

Dr. Bogun was supported by a grant from the Deutsche Forschungsgemeinschaft.

Address for correspondence: Fred Morady, M.D., University of Michigan Medical Center, 1500 East Medical Center Dr., B1F245, Ann Arbor, MI 48109-0022. Fax: 313-936-7641.

Manuscript received 8 August 1995; Accepted for publication 18 September 1995. ment of recurrent VT with a rate of 240 beats/min, a right bundle branch block configuration, and right-axis deviation. The patient experienced symptoms of rapid palpitations, lightheadedness, chest discomfort, and weakness during episodes of VT; there was no history of syncope. When VT first occurred 5 years earlier, he was treated with $480 \mathrm{mg} /$ day of verapamil, which suppressed recurrences of VT for 1 year. However, in the 4 years prior to referral, nine episodes of sustained VT necessitating visits to an emergency room for treatment with intravenous verapamil or electrical cardioversion occurred. In the year prior to referral, he was treated with $540 \mathrm{mg} /$ day of verapamil, with three recurrences of sustained VT. These episodes of VT all had the same configuration: right bundle branch block with right-axis deviation. The physical examination and ECG during sinus rhythm were normal, and there was no evidence of structural heart disease detected by echocardiography or coronary angiography.

After informed consent was obtained, an electrophysiologic procedure was performed 48 hours after discontinuation of therapy with verapamil. 
Pacing was performed at twice threshold with stimuli $2 \mathrm{msec}$ in duration. Electrogram recordings were filtered with a bandpass of 50 to $500 \mathrm{~Hz}$. Right ventricular overdrive pacing at cycle lengths of 290 to $230 \mathrm{msec}$ reproducibly induced VT with a cycle length of $265 \mathrm{msec}$ and a right bundle branch block configuration with right-axis deviation, identical to the spontaneous episodes of VT (Fig. 1).

A deflectable 7-French quadripolar electrode catheter with a 4-mm tip electrode and 2-5-2 mm spacing between electrodes was introduced into the right femoral artery and advanced into the left ventricle using a retrograde aortic approach. Pace mapping and endocardial activation mapping were performed. The earliest endocardial activation occurred 20 msec before the onset of the QRS complex and was found at the anterobasal portion of the left ventricle, approximately $2 \mathrm{~cm}$ from the aortic valve (Fig. 1). Pacing at a cycle length similar to the VT cycle length at this site resulted in QRS complexes that were very similar or identical to the VT QRS complexes in each of the 12 ECG leads (Fig. 1). Ap- plications of radiofrequency energy at this site were ineffective in terminating VT despite adequate tissue healing as judged by impedance monitoring. ${ }^{3}$

At several sites within an area of $1 \mathrm{~cm}^{2}$ on the anterobasal wall of the left ventricle adjacent to the site at which the earliest endocardial activation was present, a sharp deflection that preceded the QRS complex by 10 to $30 \mathrm{msec}$ was recorded during both sinus rhythm and VT (Fig. $2 \mathrm{~A})$. At the site where this potential was the earliest, endocardial ventricular activation during VT occurred simultaneously with the onset of the QRS complex (Fig. 2A). Pace mapping at this site demonstrated similar QRS complexes during VT and pacing in only 1 of the 12 ECG leads (Fig. 2B). A 60-second application of $18 \mathrm{~W}$ of radiofrequency energy at this site was effective in terminating VT within 2 seconds and preventing its reinduction. The high-frequency potential recorded at the target site remained unchanged after ablation of the VT. The ECG during sinus rhythm remained normal, without right-axis de-

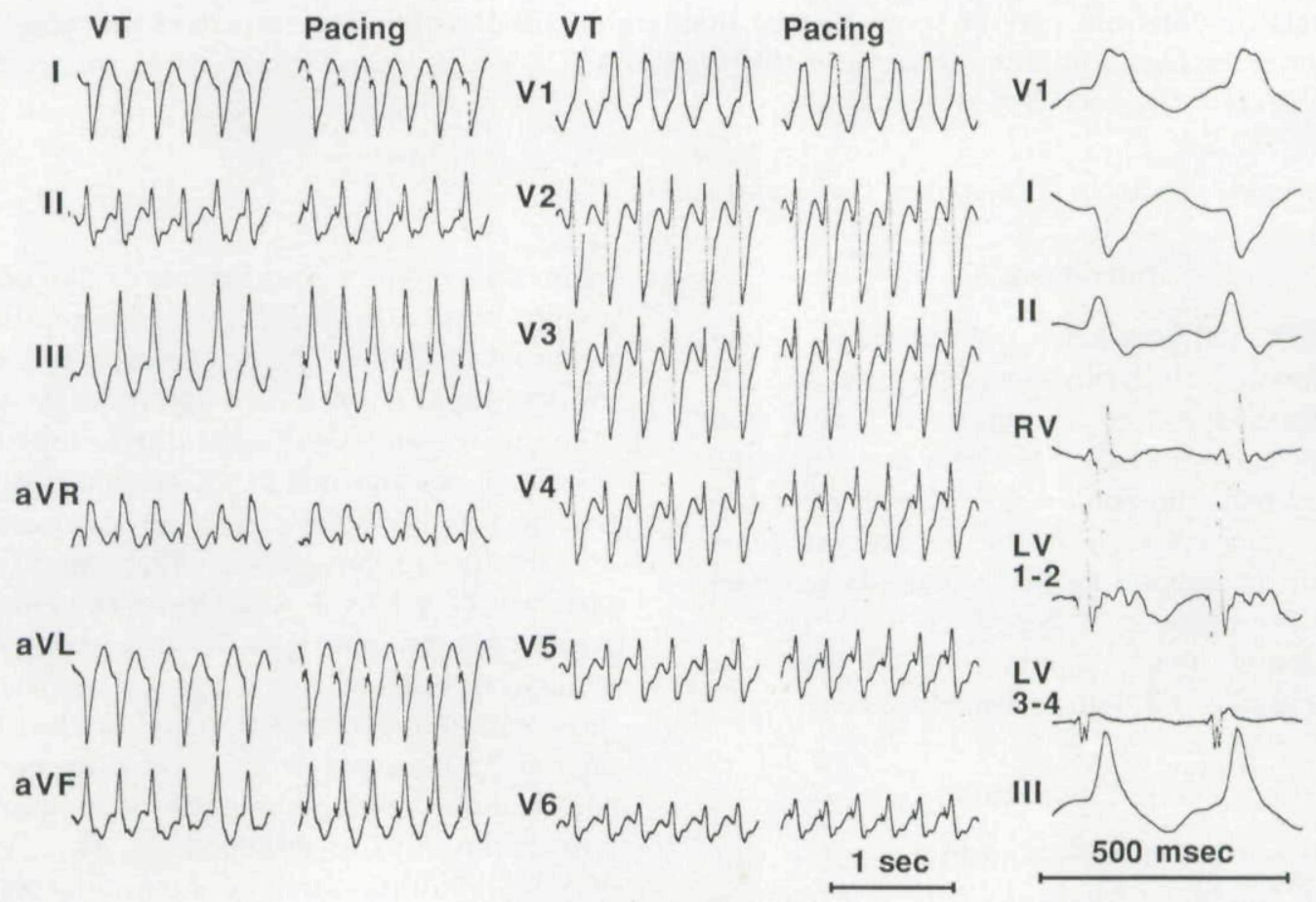

Figure 1. Twelve-lead ECG of the ventricular tachycardia (VT) induced in the electrophysiology laboratory. The VT had a cycle length of $265 \mathrm{msec}$ and was identical in appearance to the patient's spontaneous episodes of VT. Also shown are the QRS complexes generated by pacing at a cycle length of $250 \mathrm{msec}$ at the anterobasal left ventricle, $2 \mathrm{~cm}$ from the aortic valve. On the right are shown leads VI, I, II, the right ventricular electrogram $(R V)$, the electrogram recorded by the distal pair of electrodes of the ablation catheter positioned at the anterobasal left ventricle (LV 1-2) at the same site at which the pace map was generated, the electrogram recorded by the proximal pair of electrodes of this catheter (LV 3-4), and lead III. Ventricular activation recorded by the ablation catheter (LV 1-2) preceded the QRS complexes by 20 msec. Despite the excellent pace map, catheter ablation at this site was unsuccessful. 


\section{Ventricular Tachycardia}<smiles></smiles>

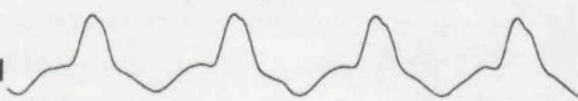<smiles>CCCCCCCCCCCCCI</smiles>

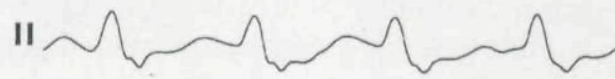

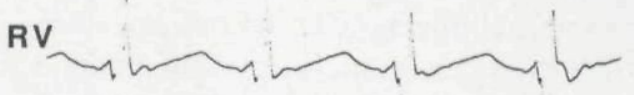

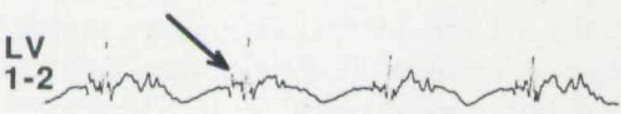

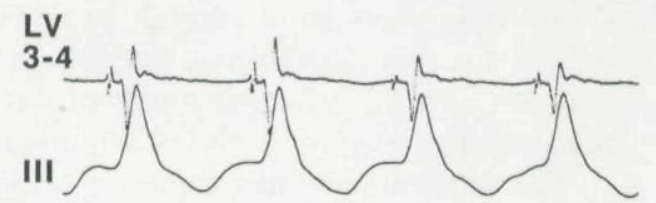

A

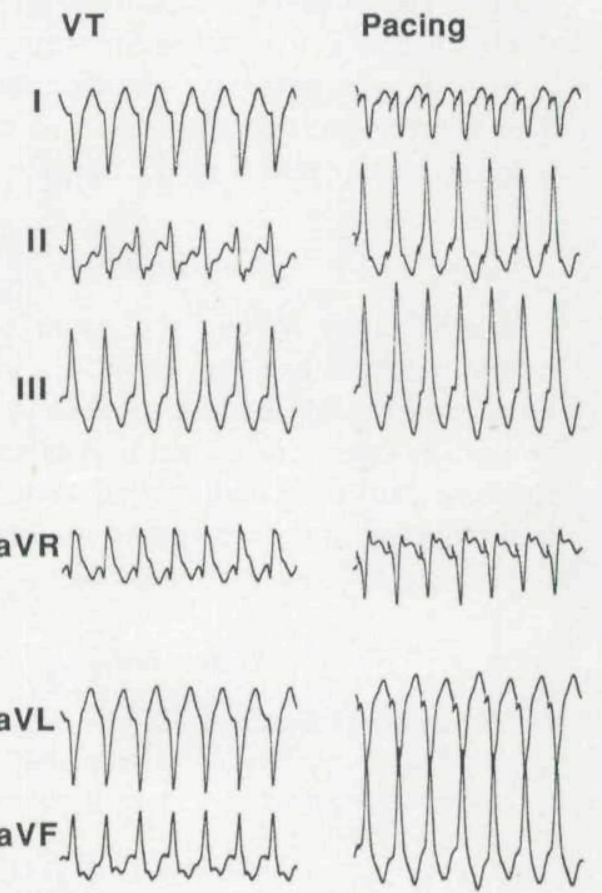

\section{Sinus Rhythm}
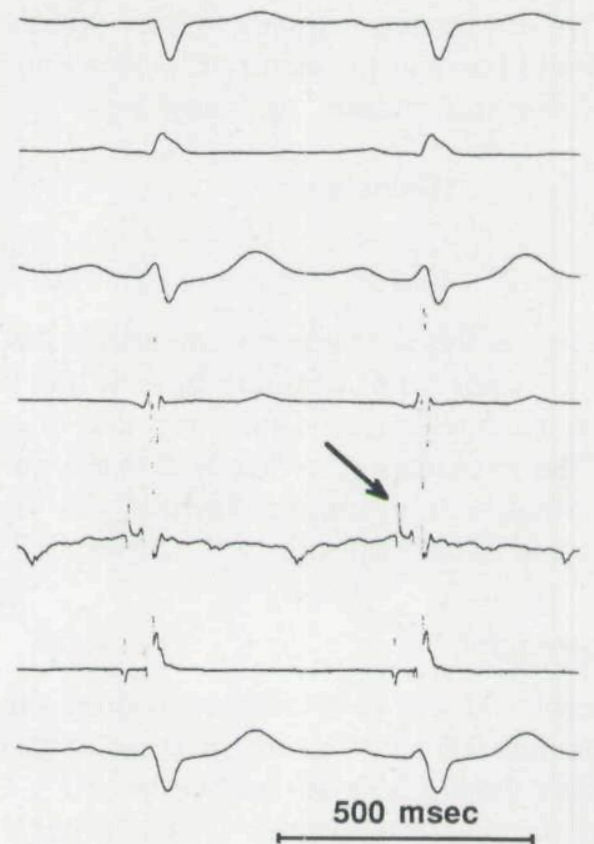

Pacing
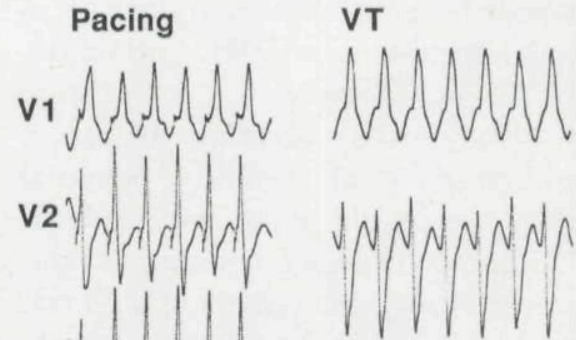

v3 y)

$24 \sqrt{4} \sin$

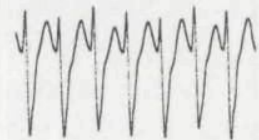

204 )

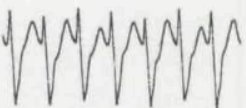

vodhldwh

B

Figure 2. The earliest presumed Purkinje potential recorded at the anterobasal left ventricle, approximately $1 \mathrm{~cm}$ from the site shown in Figure 1. Abbreviations as in Figure 1. (A) A sharp deflection was recorded during ventricular tachycardia by both pairs of electrodes of the ablation catheter. The potential preceded the QRS complexes by 30 msec. The ventricular depolarization was simultaneous with the onset of the QRS complexes. The presumed Purkinje potential also was present during sinus rhythm and preceded the QRS complexes by $30 \mathrm{msec}$. The arrows point out the presumed Purkinje potentials. (B) A comparison of QRS complexes during ventricular tachycardia (VT) and pacing at a cycle length of 250 msec at the left ventricular anterobasal site at which the presumed Purkinje potentials shown in Figure $2 A$ were recorded. The QRS complexes were similar only in lead aVL. Nevertheless, catheter ablation at this site was effective. 
viation. A follow-up electrophysiologic test 48 hours later demonstrated no inducible VT. The patient has not been treated with any antiarrhythmic medication and has had no recurrences of symptomatic VT during 6 months of follow-up.

\section{Discussion}

\section{Main Findings}

The findings in this case report demonstrate that idiopathic VT with a right bundle branch block configuration and right-axis deviation may arise from the area of the left anterior fascicle and that a potential consistent with a Purkinje potential may be useful in guiding catheter ablation of this type of VT.

\section{Purkinje Potentials}

The anterobasal left ventricular recording site and its presence 10 to $30 \mathrm{msec}$ in front of the QRS complex during both sinus rhythm and VT suggest that the potential identifying the effective ablation site was generated by the Purkinje fibers of the left anterior fascicle. Although the potential is similar in appearance to a His-bundle depolarization or left bundle potential, its location on the free wall of the left ventricle argues in favor of a Purkinje potential. A similar potential was described by Wen et al. ${ }^{4}$ in a patient with idiopathic left VT and right-axis deviation. In contrast to the present study, this potential was considered nonspecific for a successful ablation site.

Radiofrequency catheter ablation of the VT resulted neither in elimination of the presumed Purkinje potential nor the appearance of right-axis deviation during sinus rhythm. This suggests that only a small and/or distal portion of the Purkinje fibers of the left anterior fascicle were ablated.

\section{Pace Mapping}

The observation that pacing at the effective ablation site resulted in similar QRS complexes as during VT in only 1 of 12 ECG leads strongly suggests that the site within the Purkinje network at which the VT originated did not correspond with the site at which global ventricular depolarization was initiated during VT. As has been previously suggested in cases of VT originating in the left posterior fascicle, ${ }^{1}$ this finding implies that the Purkinje fibers generating the VT are insulated from the immediately adjacent myocardium. As postulated by Nakagawa et al., ${ }^{1}$ the myocardium may be activated as an "innocent bystander," with sub- sequent conduction of the VT wavefront from a Purkinje-myocardial junction back to the myocardium adjacent to the fibers generating the earliest Purkinje potential; this would account for the later endocardial activation time at the ablation site.

In the study by Nakagawa et al., ${ }^{1}$ pacing at the effective target site in 4 of 8 patients with left posterior fascicular VT resulted in QRS complexes similar to the VT complexes, with a stimulus-QRS interval identical to the Purkinje potential-QRS interval during VT. In contrast, in the present report, pacing at the successful ablation site resulted in QRS complexes that differed significantly from the VT complexes. This suggests that the pacing stimuli (which were twice diastolic threshold) were strong enough or the distal electrode (which was $4 \mathrm{~mm}$ long) was large enough to result in capture of not only the Purkinje fibers generating the VT, but also the adjacent myocardium that was not directly involved in the generation of VT,

The target site at which there was a close match between pacing and VT and at which the earliest endocardial activation was recorded presumably was at the exit site of the VT wavefront into the myocardium. Of note is that radiofrequency energy applied at this site was ineffective despite adequate heating. This suggests that the exit site may have been broader than the lesion created by the ablation catheter.

\section{Conclusion}

Idiopathic VT with a right bundle branch configuration and right-axis deviation may originate from the area of the left anterior fascicle. A potential presumed to represent a Purkinje potential may be more helpful than endocardial ventricular activation mapping or pace mapping in identifying an effective target site for ablation of this type of VT.

\section{References}

1. Nakagawa H, Beckman KJ, McClelland JH, et al: Radiofrequency ablation of idiopathic left ventricular tachycardia guided by a Purkinje potential. Circulation 1994;88:2607-2617.

2. Ohe T, Shimomura K, Aihara N, et al: Idiopathic sustained left ventricular tachycardia: Clinical and electrophysiologic characteristics. Circulation 1988;77: 560-568.

3. Strickberger SA, Ravi S, Daoud E, et al: The relationship between impedance and temperature during radiofrequency ablation of accessory pathways. Am Heart J (In press).

4. Wen MS, Yeh SY, Wang CC, et al: Radiofrequency ablation therapy in idiopathic left ventricular tachycardia with no obvious structural heart disease. Circulation 1994;89:1690-1696. 
This document is a scanned copy of a printed document. No warranty is given about the accuracy of the copy. Users should refer to the original published version of the material. 